\title{
RELATIVISTIC MANY-ELECTRON SCF CORRELATION DIAGRAM FOR $\mathrm{Pb}-\mathrm{Pb}$
}

\author{
B Fricke, W D Sepp and T Morović
}

Department of Physics, University of Kassel

3500 Kasse1, West Germany

Correlation diagrams play an important role in the physical understanding and interpretation of colliding atomic systems. In the case of high-energy heavy-ion collisions they have amply shown their validity, as - for examples - in calculation of the well-known $2 \mathrm{p}_{\pi}-2 \mathrm{p}_{\sigma}$ rotational coupling ${ }^{1}$, interpretation of MO $\mathrm{x}$-rays ${ }^{2}$, or just the empirical connection of anisotrcpy maxima in the Mo x-rays and minima in the correlation diagram ${ }^{3-4}$. With a discrete variational method $^{5}$ we have developed a special program to calculate diatomic molecules in a fully relativistic regime, using numerical atomic Dirac-Fock-Slater basis functions of the two colliding atoms as well as of the Z-united atom at the center of gravity. After prediagonalization we are able to calculate, with a self-consistent charge procedure, correlation diagrams of very heavy systems. In Figure 1 we present the correlation diagram of $\mathrm{Pb}$ on $\mathrm{Pb}$ with 96 electrons. A careful comparison with the one-electron correlation diagram given by Kirsch et $a 1^{6}$ shows a number of significant differences and physically important changes:

(a) Even the strongest bound electronic levels are considerably screened. At $R=50 \mathrm{fm}$ the binding is lower by $8 \%$ for the $1(1 / 2) \mathrm{g}$, $25 \%$ for the $1(1 / 2)_{\mathrm{u}}$ and $40 \%$ for the $3(1 / 2) \mathrm{g}$ level. By $\mathrm{R}=500 \mathrm{fm}$ these changes become respectively $13 \%, 34 \%$ and $50 \%$.

(b) Due to the change in screening at small internuclear distances the lowest levels, which correlate to $j=1 / 2$ atomic levels, decrease even steeper at $\mathrm{R} \rightarrow 0$ when compared with the oneelectron correlation diagram. 
(c) The $1(1 / 2)_{u}$ leve1 (which non-relativistically is called 2po) shows a very pronounced minimum at $R \approx 2000 \mathrm{fm}$, the energy eigenvalue being about $120 \mathrm{keV}$. This minimum does not exist in the one-electron correlation diagram given by Kirsch et $a 1^{6}$. It shows only a region where the $2 \mathrm{p}_{\sigma}$ level is relatively constant in energy. This clear minimum in the many-electron correlation diagram is very important since Stoller et $a 1^{7}$ observe an anisotropy maximum near $130 \mathrm{keV}$ in $\mathrm{Pb}-\mathrm{Pb}$ collisions. According to the interpretation given by Gippner et $\mathrm{a} 1^{3}$ and Stoller et a $1^{4}$ such anisotropy maxima are strongly correlated with the minima in the correlation diagram. The existence of the $2 \mathrm{p}_{\sigma}$ minimum is therefore a strong support for this interpretation, which seems also to be valid in the region of such heavy systems.

(d) At sma11 internuclear distances, of $\sim 500 \mathrm{fm}$, our many-electron correlation diagram shows pronounced minima for many levels correlating with atomic levels with $j>1 / 2$.

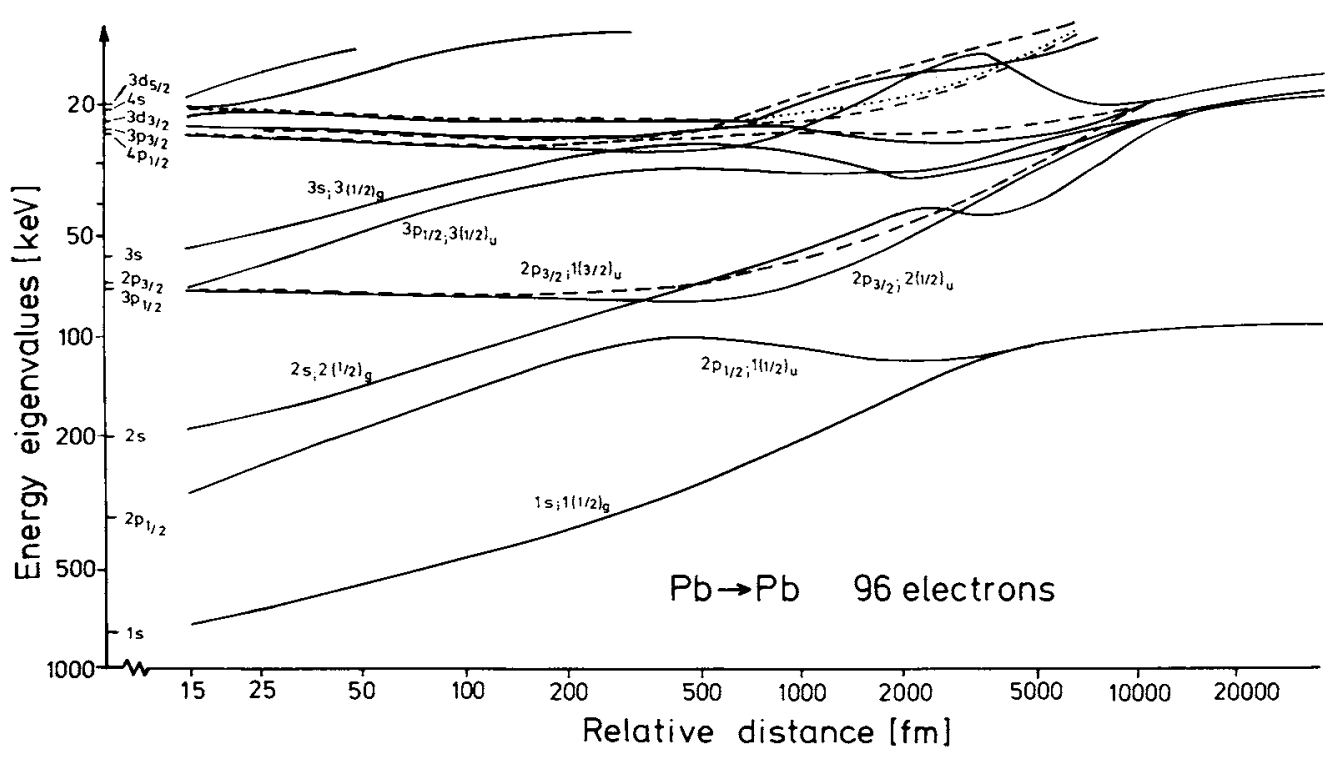

Fig. 1 Self-consistent-charge relativistic Dirac-Fock correlation diagram for $\mathrm{Pb}$ on $\mathrm{Pb}$ with 96 electrons. The levels are classified on the left according to the z-united atomic notation; while on the curve the relativistic molecular nomenclature is indicated. 
(e) The 'forbidden' crossing between the 1(1/2) and $2(1 / 2)_{\mathrm{u}}$ levels is located at $R \approx 500 \mathrm{fm}$ with an energy difference $\Delta \mathrm{E} \approx 20 \mathrm{keV}$. The corresponding values from the 1-electron correlation diagrams are $R \approx 700 \mathrm{fm}$ and $\Delta \mathrm{E} \approx 35 \mathrm{keV}$. A coupled-channel calculation shows an increase of up to nearly an order of magnitude of the hole transfer when going from the one-electron levels to the level-structure in Figure 1.

(f) Due to the strong increase in binding energy for all levels correlating with atomic levels with $j=1 / 2$ at very small internuclear distances, a non-negligible proportion of holes could be transferred from the $M-s h e 11$ via the L-shell into the K-shell of $\mathrm{Pb}$ atoms in a single collision process. This is possible due to radial coupling between the $3(1 / 2)$ u level and the levels correlating with the M-shell. At very small distances the holes can be transferred from the $3(1 / 2)_{\mathrm{u}}$ level to the $2(1 / 2)_{\mathrm{u}}$ and $1(3 / 2)_{u}$ levels by respectively radial and rotational coupling. Finally, the crossing between the $2(1 / 2)_{u}$ and $1(1 / 2)_{u} 1$ evels as discussed in (e) will allow a large hole transfer into the $\mathrm{K}-$ shell of $\mathrm{Pb}$. This coupling increases for even heavier systems because of the increase of the $3 p_{1 / 2}$ z-united binding. This may be a possible explanation for the unexplained increase of the $1 s_{\sigma}$ ionization in the system $\mathrm{Pb}-\mathrm{Cm}^{8}$.

\section{REFERENCES}

1. K Taulbjerg and J S Briggs, J. Phys. B8, 1895 and 1909 (1975)

2. B Fricke, T Morović, $W-D$ Sepp, A Rosén and D E Ellis, Phys. Lett. 59A, 375 (1976)

3. P Gippner, $\mathrm{K}-\mathrm{H}$ Kaun, $\mathrm{H}$ Sodan, F Stary, W Schulze and Yu P Tretyakov, Phys. Lett. 60B, 249 (1976)

4. Ch Stoller, W Wölfli, G Bonani, M Stöckli and M Suter, J. Phys. B10, L347 (1977)

5. See for the general method: A Rosén and D E Ellis, J. Chem. Phys. 62, 3039 (1975)

6. J Kirsch, W Betz, J Reinhardt, G Soff, B Müller and W Greiner, Phys. Lett. 72B, 298 (1978)

7. Ch Stoller, E Morenzoni, W Wölfli, W E Meyerhof, F Folkmann, $T$ Vincelt, $\mathrm{P} H$ Mokler and P Armbruster, Z. Physik (to be published)

8. D Liesen, P Armbruster, F Bosch, S Hagmann, P H Mokler, $\mathrm{H}-\mathrm{J}$ Wollersheim, $\mathrm{H}$ Schmidt-Böcking, R Schuch and J B Wilhelmy, Phys. Rev. Lett. 44 , 983 (1980) 